\title{
ON THE PERFORMANCE OF INELASTICALLY RESPONDING ASYMMETRIC STRUCTURES DESIGNED BY THE MODAL RESPONSE SPECTRUM METHOD OF NZS 4203:1992
}

\author{
A. M. Chandler ${ }^{1}$ and X. N. Duan ${ }^{2}$
}

\begin{abstract}
The primary objective of this paper is to evaluate the effectiveness of the 3-dimensional modal response spectrum method (MRSM), as specified in the seismic design regulations of NZS 4203:1992, in accounting for torsional effects arising in stiffness-asymmetric buildings. Such buildings are assumed to be excited well into the inelastic range of response, where ultimate limit state design criteria are applicable. Also included is a study of the horizontal regularity conditions of NZS 4203:1992. This preliminary study focuses on the influence that these regularity conditions have on the selection of an appropriate codified approach for the design of torsionally asymmetric buildings. In particular, restrictions on the use of the equivalent static method of design (ESM), leading to a requirement to employ the MRSM to account for dynamic torsional effects, are discussed in some detail. The dynamic response studies indicate that in systems designed by the MRSM, no significant increase in ductility demand arises in flexible-edge elements (compared with symmetric systems). Furthermore, the MRSM may be over-conservative for the design of flexible-edge elements, in cases of structures having intermediate or large eccentricity. However, significant additional ductility demand may arise in the stiff-edge element in highly asymmetric buildings subjected to strong ground motion. In such cases, therefore, structures designed by the MRSM may not achieve the design aim of consistent protection given to symmetric and asymmetric systems against excessive inelastic response and consequent structural damage.
\end{abstract}

\section{INTRODUCTION AND OBJECTIVES}

It is widely accepted that the two main objectives of earthquake-resistant design are to ensure safety of life and to protect property. With reference to asymmetric buildings, termed more generally torsionally unbalanced buildings, these objectives require firstly that in serviceability limit state design, element strengths are specified which are sufficiently high to ensure elastic behaviour when the building is subjected to small or moderate ground motions, hence limiting deformations and consequent damage. Secondly, in satisfying ultimate limit state design criteria it is expected that in severe earthquakes no significant additional ductility demand should arise in the earthquake load-resisting elements (columns and/or walls), compared with corresponding symmetric or torsionally balanced systems responding only in translation.

To satisfy the above criteria, seismic building codes include various forms of torsional design provisions. It is evident when reviewing such provisions that current design practice for torsional effects, whether using the MRSM or the ESM, is based largely on linear (or equivalent linear) elastic theory. In general, a non-linear inelastic analysis is not required by present code regulations, even for buildings with torsional irregularities, although it may be recommended in some special cases. This paper addresses the question of the influence that non-linear hysteretic behaviour due to yielding of the resisting structural elements has on the torsional effect in asymmetric buildings. Focus is placed on the MRSM of NZS 4203:1992 [1], as an example of the extrapolation of procedures based largely on elastic design criteria to the inelastic design of buildings exhibiting significant torsional effects. The main objectives (in the order in which they are considered in the paper) are (a) to examine analytically the horizontal regularity conditions of NZS 4203:1992, and to discuss their logic in the context of providing restrictions on the use of the ESM to design for torsional effects, (b) to assess whether or not designs based on the MRSM provide reasonably uniform protection to resisting elements situated on the flexible and stiff sides of stiffness-asymmetric structures, and (c) to determine whether the MRSM provides consistent control over inelastic structural response (and hence damage) for both symmetric and asymmetric systems.

\section{ANALYTICAL BUILDING MODEL}

The effectiveness of the MRSM has been assessed in later sections, with reference to a 3-element single storey stiffness-asymmetric building model with a rigid floor diaphragm (Figure 1). The rectangular structure has a plan aspect ratio, $\lambda(=b / a)$. The lateral load-resisting elements are

1 Reader in Earthquake Engineering, Department of Civil and Environmental Engineering, University College London (Member)

Research Fellow, Department of Civil and Environmental Engineering, University College London, London, U.K. 


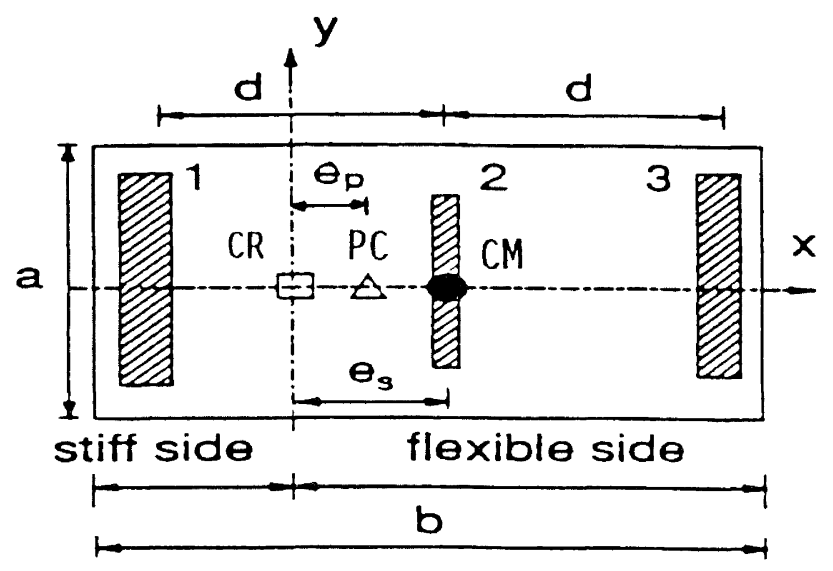

$$
\begin{aligned}
& C R=\text { Floor Centre of Rigidity } \\
& C M=\text { Floor Centre of Mass } \\
& P C=\text { Storey Plastic Centroid }
\end{aligned}
$$

Figure 1. Asymmetric floor plan configurations in the selected 3-element model.

assumed to be bi-linear with 3 per cent strain hardening. A (static) eccentricity, $e_{s}$ is assumed, measured between the floor centres of mass and rigidity, $\mathrm{CM}$ and $\mathrm{CR}$, respectively. The centres of rigidity, which in general are load-dependent quantities, are defined [2] as the set of points through which the lateral earthquake loading should be applied in order to result in no rotation at any floor level, relative to the building's base. For a building responding only in translation, the position of the resultant of the element shear forces in any storey (for example, elements 1 to 3 in Figure 1, resisting forces in the lateral y-direction), defines the location (in plan) of the storey shear centre. In single storey buildings, or multistorey buildings with idealised rigid floor diaphragms and proportional framing systems, the storey shear centre(s) lie on the same vertical axis as the floor centre(s) of rigidity, CR [3].

The parameter $\Omega$ is defined as the ratio of torsional to translational (lateral) fundamental frequencies in equivalent symmetric systems. For a defined mass distribution, $\Omega$ is a measure of a structure's torsional stiffness or capacity to resist torsional deformation. This parameter has been extensively employed in studies of torsional coupling effects in both the elastic and inelastic ranges of seismic building response [4-9]. The element spacing, d, (with the elements distributed symmetrically about CM) is determined such that $\Omega$ has a fixed selected value, taken to be 1.0 for the purposes of the dynamic analyses presented below. A series of related studies of the torsional response of parametrically-defined buildings [6-8] concluded that the inelastic response of torsionally stiff asymmetric buildings $(\Omega>1.2)$ is only slightly smaller than that of buildings with intermediate torsional stiffness $(\Omega \approx 1.0)$. The insensitivity of the dynamic inelastic response to $\Omega$, when $\Omega \geq 1.0$, is in direct contrast to the torsionally coupled elastic response of asymmetric buildings [4],[5]. Hence in the inelastic range, the model with $\Omega=1.0$ provides conservative results for buildings with intermediate or high torsional stiffness, as encouraged by codes. Therefore, the adoption of this value does not sacrifice the generality of the inelastic dynamic results presented herein.
The building is assumed to have viscous damping equal to $5 \%$ of critical damping in each of its two coupled modes of vibration, a value which is appropriate for the types of structure represented by the adopted model and which is consistent with the code design spectra for the MRSM

It is further assumed that resisting elements 2 and 3 have identical elastic stiffnesses $\left(k_{2}=k_{3}\right)$. The stiffness eccentricity $e_{s}$ is therefore introduced by increasing the stiffness of element 1 , compared with that of a symmetric reference model with uniform element properties (stiffness and strength). The symmetric reference model is employed below in assessing the inelastic dynamic response of buildings with stiffness asymmetry. The assumption that $k_{2}=k_{3}$ is representative of a class of structures with moderate torsional stiffness $[10]$, as appropriate for the selected case $\Omega=1.0$. The position of the resultant of the element design strengths defines the storey centre of strength or plastic centroid, PC (Figure 1), and hence the corresponding strength eccentricity, $\mathrm{e}_{\mathrm{p}}$, the latter measured to the right of $\mathrm{CR}$.

\section{DISCUSSION OF CODE HORIZONTAL REGULARITY REQUIREMENTS}

NZS 4203:1992 specifies horizontal regularity requirements, to define limits for the application of the ESM in designing for seismic torsional effects. In this study which focuses primarily on structures designed by the MRSM, it is important first to establish what criteria lead to its use in designing for torsion in asymmetric-plan buildings. The static torsional provisions of NZS 4203:1992, as employed in the ESM, are the subject of a separate investigation [11]

NZS 4203:1992 specifies that for a structure to be horizontally regular, the diaphragms should not contain abrupt variations in stiffness or major re-entrant corners such as could significantly influence the distribution of the lateral forces in the structure. In addition, with regard to torsional effects, horizontal regularity implies that the building under consideration has either a small eccentricity (of stiffness and/or mass), or a high torsional stiffness. The latter requires that the parameter $\Omega$, as defined above, has a relatively high value. Hence, NZS 4203:1992 states that either (but not necessarily both) of the following two criteria specified in Clause 4.4.1(a) should be satisfied. Firstly, criterion (i) states that the horizontal distance from the shear centre at any level to the CM for all levels above, should be less than 0.3 times the plan dimension, b. In the example structure shown in Figure 1, this distance is simply equivalent to the eccentricity, $e_{s}$. Alternatively, the second criterion (ii) states that under the application of the equivalent static design loading at a point $0.1 \mathrm{~b}$ from $\mathrm{CM}$, at each floor level (Figure 2), the following edge displacement condition should apply:

$$
y_{2} \leq(7 / 3) y_{1}
$$

Equation (1a) can also be expressed as:

$$
\left(y_{2}-y_{1}\right) \leq 0.4\left(y_{1}+y_{2}\right)
$$

or

$$
\left.y_{2} \leq(7 / 5)\left[y_{1}+y_{2}\right) / 2\right]
$$

The intention of criterion a(ii) defined in eqns. (1a) to (1c) is that, for structures with a given plan eccentricity, $e_{s}$, sufficient torsional stiffness should be provided in the layout of the 


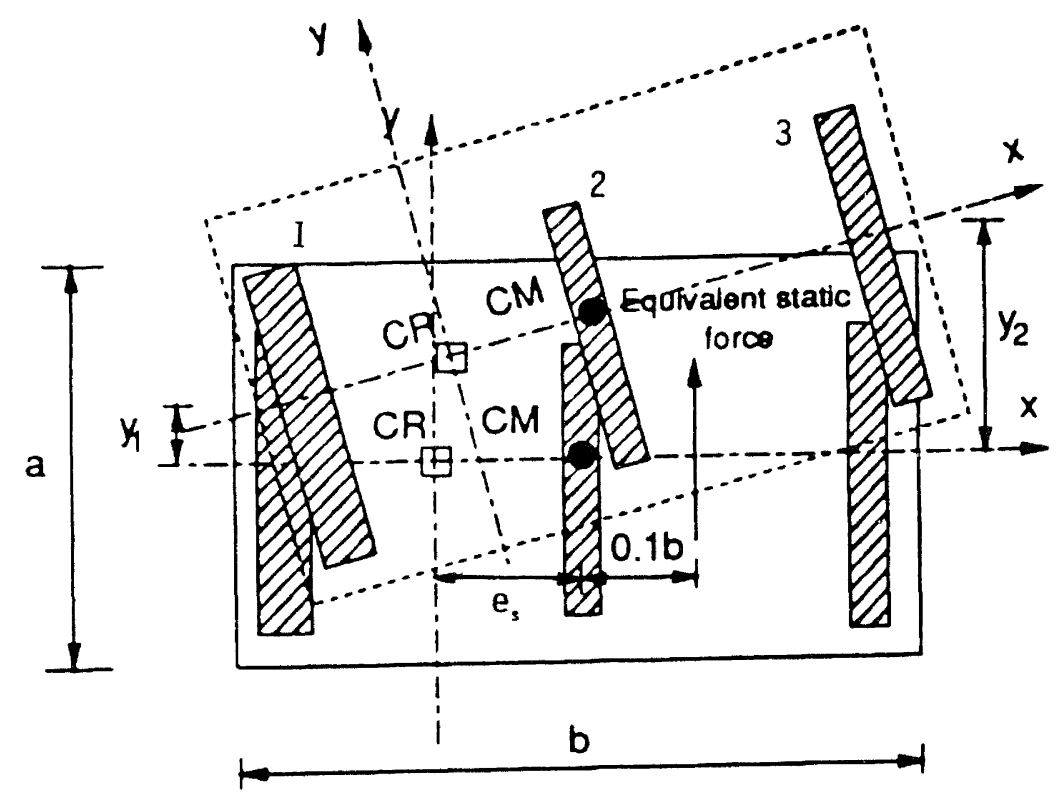

Horizontal regularity requirements are either $e_{s} / b<0.3$ or $y_{2} \leq(7 / 3) y_{1}$

$$
\begin{aligned}
\delta & =\text { displacement ratio } \\
& =\left(y_{2}-y_{1}\right) /\left(y_{1}+y_{2}\right) \\
\lambda & =\text { plan aspect ratio }=(b / a)
\end{aligned}
$$

Figure 2. Definition of alternative horizontal regularity criteria of NZS 4203:1992.

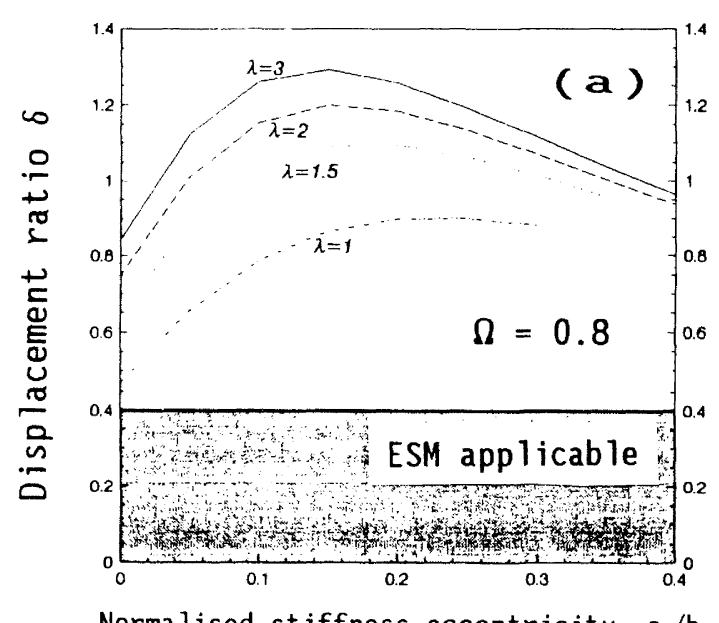

Normalised stiffness eccentricity $e_{s} / b$
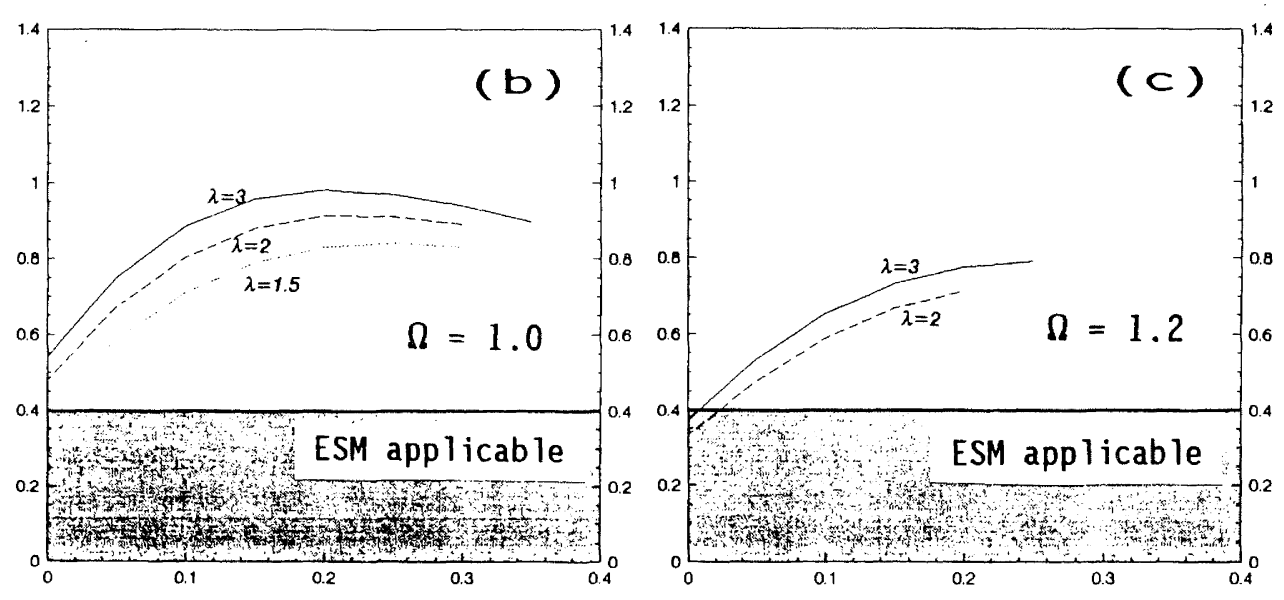

Normalised stiffness eccentricity $e_{2} / b$

Normalised stiffness eccentricity $e_{3} / b$

Figure 3. Evaluation of the horizontal regularity criterion a(ii) of Clause 4.4.1 of NZS 4203:1992. 
resisting elements (frames and shear walls) to prevent excessive floor rotation. Interpreting eqns.(1b) and (1c) implies that in the former case, the difference in the displacement of the two sides of the structure at any level in the direction of loading does not exceed $40 \%$ of the sum of the lateral displacement of the two sides, in the same direction. Alternatively, in the latter case the horizontal regularity criterion is expressed as a condition that the peak lateral edge displacement, $y_{2}$ (Figure 2), should not be more than 1.4 times the average of the edge displacements, $y_{1}$ and $y_{2}$. Note that the displacement $y_{2}$ relates to the so-called flexible edge of the stiffness-asymmetric structure illustrated in Figure 1 , and correspondingly, $y_{1}$ relates to the stiff edge.

To examine the form of restriction imposed by the second of the above horizontal regularity criteria [a(ii) in Clause 4.4.1], an evaluation has been made for asymmetric buildings with a range of stiffness eccentricities $e_{s}$ (normalised to $b$ ), and for plan aspect ratios, $\lambda(=b / a)$, in the range 1 to 3 . The results have been plotted in Figure 3, where the displacement ratio, $\delta$, is defined in relation to Eqn.(1b) as:

$$
\delta=\frac{\left(\mathrm{y}_{2}-\mathrm{y}_{1}\right)}{\left(\mathrm{y}_{1}+\mathrm{y}_{2}\right)}
$$

Hence, according to NZS 4203:1992, the torsional design provisions of the ESM are applicable in cases where $\delta \leq 0.4$, as shown in Figure 3. Note that the first horizontal regularity criterion a(i), limiting the eccentricity, $e_{s}$, to $0.3 b$, is an independent condition, as discussed below. The presented results indicate that buildings in which the torsional response is most significant, namely those having intermediate or large eccentricity combined with low or intermediate torsional stiffness (the latter represented by $\Omega=0.8$ or 1.0 , respectively $[12,13]$, see Figure 3(a),(b)), will in all cases fail to satisfy this horizontal regularity criterion, irrespective of the values of the eccentricity, $e_{s}$, or the aspect ratio, $\lambda$. Nevertheless, provided $\mathrm{e}_{\mathrm{s}}<0.3 \mathrm{~b}$, the first horizontal regularity criterion is satisfied, implying that such buildings should be satisfactorily designed by the static torsional provisions of the ESM, as evaluated in a related study [11].

A limiting value on $\Omega$ is reached when $d=0.5 b$ (Figure 1 ), that is when the outer elements 1 and 3 are located at the stiff and flexible edges of the structure, respectively. This geometrical constraint limits $\Omega$ to values less than about 1.2 , for buildings with aspect ratio $\lambda \geq 2$, and the upper limit of $\Omega$ is even smaller for buildings with $\lambda<2$. Horizontal regularity criterion a(ii) is illustrated in Figure 3(c) for the limiting case $\Omega$ $=1.2$, where it is observed that the condition $\delta \leq 0.4$ is satisfied only for a narrow range of structures having very small values of the normalised eccentricity $e_{s} / b$ (less than about 0.03 ). Again, this criterion is seen to be much more restrictive than the criterion a(i), namely $\mathrm{e}_{\mathrm{s}}<0.3 \mathrm{~b}$.

The above results have been obtained from a simplified but nevertheless representative analysis, based on the defined building model. This analysis implies that the criterion a(ii), which is intended to limit floor rotations, does not permit any structures to be designed for torsional effects by the ESM that would be excluded by criterion a(i). Hence it is concluded that criterion a(ii), which in its present form is much more restrictive than the first criterion, is effectively redundant for most, if not all, practical cases. By introducing the horizontal regularity criteria, it is the stated intention of NZS 4203:1992 that those structures critically affected by torsion should be required to be designed by the 3-D MRSM. However, based on the above evaluation of these criteria, this does not appear necessarily to be the case. This is therefore considered to be an area worthy of further consideration by the code-drafting authorities.

\section{MODAL RESPONSE SPECTRUM METHOD (MRSM)}

The remainder of this study evaluates, on the basis of the simplified structural models defined above, the application of the 3-D MRSM to the design of asymmetric systems for inelastic (ultimate limit state) seismic torsional effects. The MRSM as specified by NZS 4203:1992 can be applied without restriction to the design of buildings; that is, it may be applied even in cases when the horizontal regularity criteria are not satisfied, whereas the ESM can only be used to account for torsional effects in the case of buildings that satisfy such criteria.

\section{Treatment of code accidental eccentricity}

When applying the MRSM, NZS 4203:1992 also stipulates that accidental eccentricity effects be accounted for, by appropriate displacement of $\mathrm{CM}$ at each floor level $( \pm 0.1 \mathrm{~b})$ from its nominal position. The codified accidental eccentricity component, $0.1 \mathrm{~b}$, arises from a series of non-quantifiable effects including all uncertainties in design which may result in additional torsional effects, as well as the rotational component of the ground motion [14]. In normal circumstances, none of the effects causing accidental eccentricity are required to be considered explicitly in design, since they are catered for by the constant eccentricity component, $\pm 0.1 \mathrm{~b}$. Correspondingly, they have been omitted from the dynamic analyses presented below. This procedure has been shown to be justified for analyses of this type [14],[15], provided consistency is maintained by omitting accidental torsional effects from the determination of element strengths in designing both the symmetric (reference) models and torsionally asymmetric cases. It should be noted that this has not always been the case in previous studies of elastic and inelastic torsional effects in code-designed buildings.

A full discussion of the treatment of code accidental eccentricity in the context of analytically-based studies of inelastic torsional effects is beyond the scope of this paper, and has been considered in detail in related studies [15],[16]. It suffices to point out that (a) conservative results are obtained by omitting the accidental torsional provisions (ATP) in designing the analytical models, compared with their inclusion, and that (b) the argument that strict adherence to the code is necessary and desirable is spurious [15], since it would be inappropriate to do so when the analyses are incapable of modelling the effects for which the ATP are specifically intended to account.

\section{Design response spectra for evaluating the MRSM}

The design response spectrum is defined according to NZS 4203:1992, as follows:

$$
C(T)=S_{m} C h(T, 1) S_{p} R Z L_{u}
$$

in which $\mathrm{R}$ (Risk factor) and $\mathrm{L}_{\mathrm{u}}$ (Limit state factor) have both been taken as 1.0. In the former case, the value corresponds to buildings in Category IV with normal occupancy or usage. For research purposes, the Zone factor $\mathrm{Z}$ has been taken as 1.2 , this being the highest value specified in the code, implying a site with severe seismicity. The product $S_{p} Z\left(S_{p}=\right.$ Structural performance factor $=0.67$ ) is therefore 0.8 . The basic seismic hazard acceleration coefficient $\mathrm{Ch}(\mathrm{T}, 1)$ in eqn.(3) has been selected as that for site subsoil category (b) (Intermediate soil 


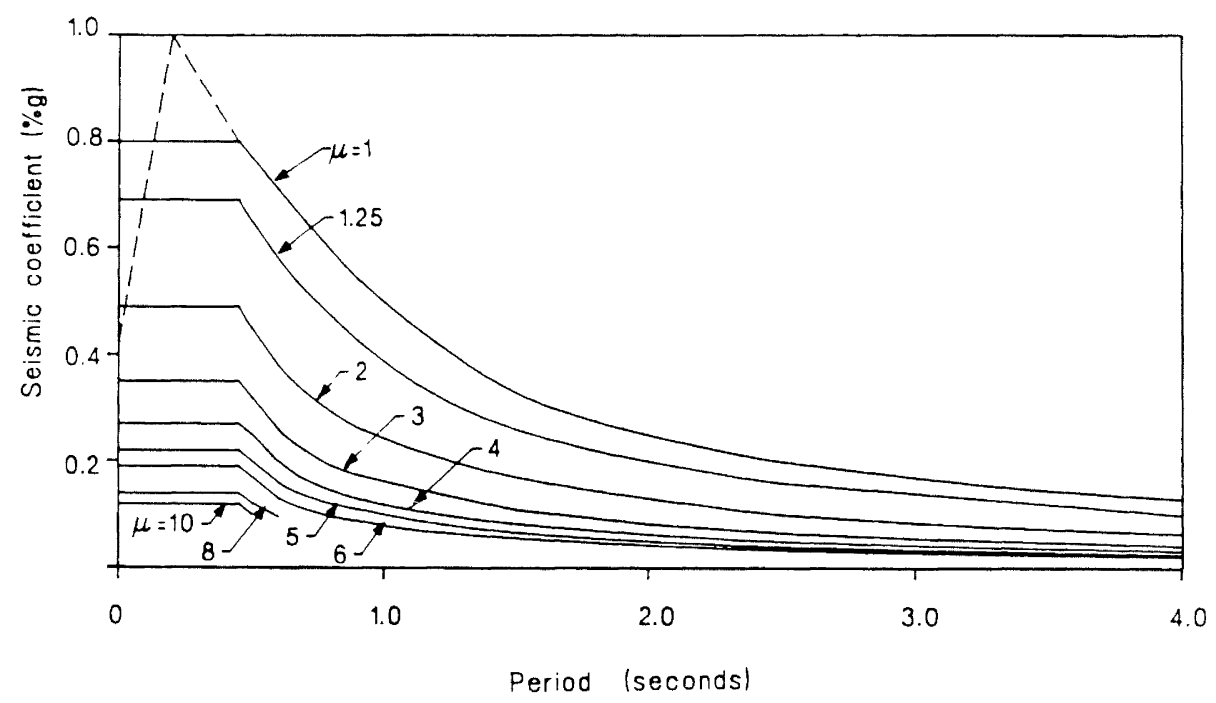

Figure 4. Basic seismic hazard acceleration coefficient of NZS 4203:1992 for intermediate soil sites $(\mu=$ Structural ductility factor $)$.

sites), illustrated in Figure 4. The two earthquake ground motions adopted in the inelastic dynamic analyses presented below have accordingly been selected from intermediate soil sites.

The design spectrum scaling factor, $\mathrm{S}_{\mathrm{m},}$ has been determined according to NZS 4203:1992, selecting $\mu$ (Structural ductility factor) as 6. $S_{\mathrm{m}}$ is the maximum of $S_{\mathrm{m} 1}$ and $S_{\mathrm{m} 2}$, where $S_{\mathrm{m} 1}$ is obtained directly from Table 4.6 .4 in the Code, taking the period $T_{1}$ to be the uncoupled lateral period, ignoring torsion, of the asymmetric systems analysed. The alternative scaling factor, $S_{\mathrm{m} 2}$, accounts for the situation in which the use of the MRSM, when the scaling factor $S_{m}=S_{m 1}$, gives a smaller design base shear than that obtained using the (inelastic) ESM This situation is commonly encountered, in particular for stiffness-asymmetric systems with a large eccentricity and relatively low torsional stiffness. For such cases, the base shear force determined using the MRSM (when $S_{m}=S_{m 1}$ ) may be as low as $50 \%$ to $60 \%$ of the inelastic base shear force determined using the (inelastic) ESM. Consequently, if horizontal regularity is assumed, the analytical results for element design strengths have been scaled up (for each mode) in cases where the combined inelastic base shear forced derived from the MRSM is less than $80 \%$ of the inelastic base shear force obtained from an equivalent static analysis $\left(\mathrm{K}_{\mathrm{m}}=0.8\right.$, where $\mathrm{K}_{\mathrm{m}}$ is the modal analysis scaling factor), such that the former is equal to the latter. For cases where horizontal regularity does not apply (see earlier discussion), the element design strengths have been scaled up (for each mode), such that the combined inelastic base shear force derived from the MRSM is equal to the inelastic base shear force obtained from an equivalent static analysis, namely taking $\mathrm{K}_{\mathrm{m}}=1.0$.

\section{ELEMENT STRENGTH DISTRIBUTION FROM THE MRSM}

\section{Element design strengths and base shear force}

The building model previously defined has been analysed by the linear elastic MRSM, employing the computer program SUPER-ETABS [17], for elastic dynamic analysis of 3-D building systems. The lateral period, $\mathrm{T}_{\mathrm{y}}$, of the reference symmetric systems has been taken over the range $0.1-2.0$ seconds, at intervals of 0.1 seconds. The static (inelastic) base shear force (SBSF) of the reference symmetric systems has been calculated according to NZS 4203:1992 and uniformly distributed to the three lateral load-resisting elements. The uncoupled lateral period of the asymmetric systems (ignoring torsion) has also been taken over the range $0.1-2.0$ seconds, at 0.1 second intervals. The elastic strength demands of the individual resisting elements of asymmetric systems have been obtained directly from the program, assuming $S_{m}=1.0$ in eqn.(3). The inelastic design strengths of individual elements are determined by scaling down their elastic strength demands, using the design spectrum scaling factor, $S_{m}$, as described in the previous section. The $\mathrm{CQC}$ rule has been employed for the modal combination.

Figure 5(a) illustrates the distribution of element design strengths (normalised to the SBSF) in direct proportion to element stiffnesses, as in stiffness and strength proportional models where no adjustment is made to account for torsional effects [10]. The distribution of element strengths, also normalised to the SBSF, in torsionally asymmetric models with allowance made for torsional effects by applying the MRSM, is shown in Figure 5(b). The results correspond to three cases, the first being a hypothetical situation in which the application of the scaling factor, $\mathrm{S}_{\mathrm{m} 2}$ (defined above), is neglected, implying that $\mathrm{S}_{\mathrm{m}}=\mathrm{S}_{\mathrm{m} 1}$ in Equation 3 for all models considered. This case has been included for reference purposes only. The second and third cases shown in Figure 5(b) correspond to $S_{m}=S_{m 2}$ with $\mathrm{K}_{\mathrm{m}}=0.8$ and 1.0 , respectively (as determined by the horizontal regularity criteria).

Figure 5(b) shows that as the eccentricity increases, with corresponding increases in the stiffness of element 1 (see the "proportional" model with stiffness and strength distributions illustrated in Figure 5(a)), the strength allocated to this element also increases, to a value of either $58 \%$ (regular) or $73 \%$ (irregular) of the SBSF, when the eccentricity ratio $\mathrm{e}_{\mathrm{s}} / \mathrm{b}=0.35$. Note, however, that these strengths are lower than in the corresponding proportional system, shown in Figure 5(a). There is a moderate decrease in the strength of element 2 (at the 
(a) Stiffness and Strength Proportional Model

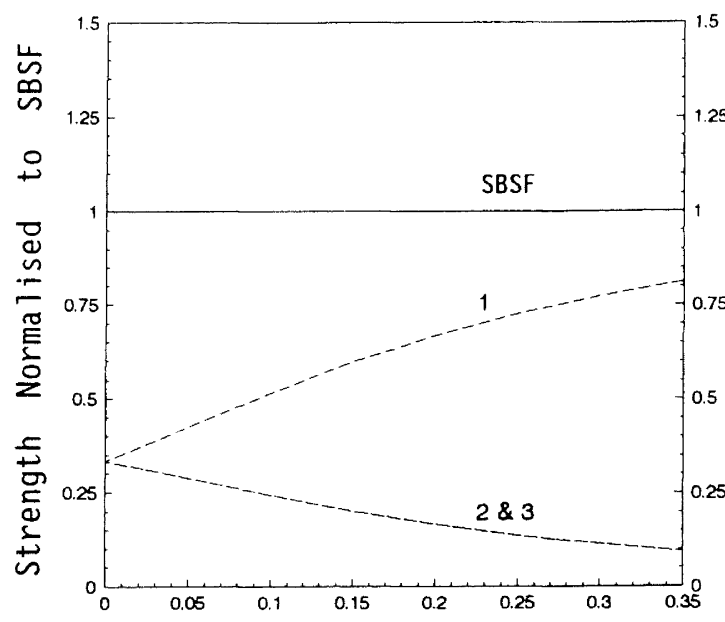

(c) BSF (MRSM)

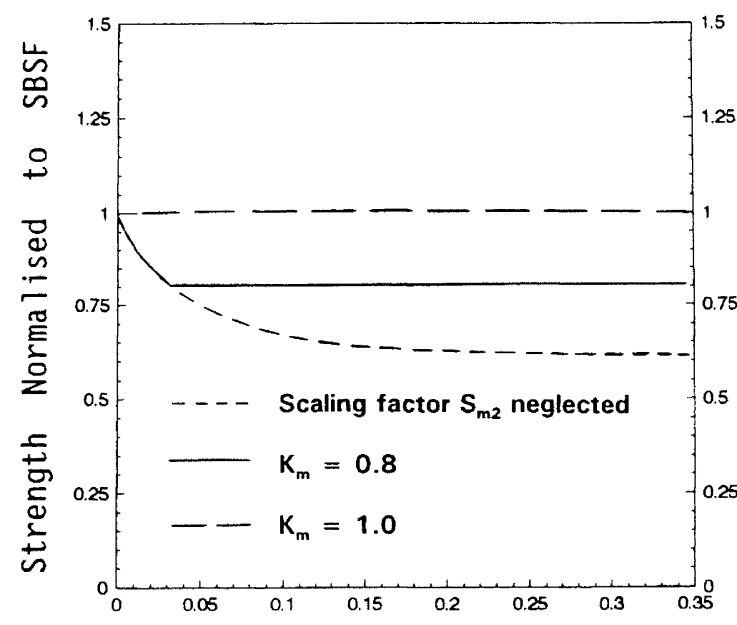

Normalised stiffness eccentricity $e_{3} / b$ (b) Torsionally Asymmetric Model (MRSM)

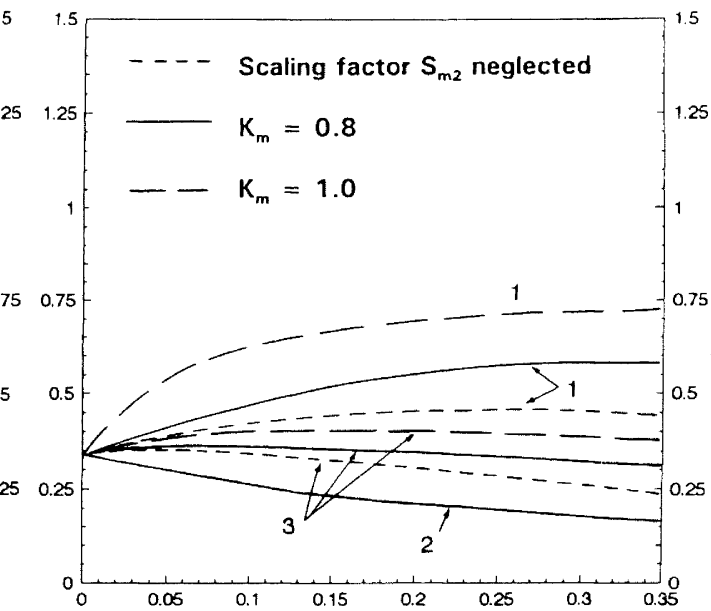

Figure 5. Variation with $e_{s}$ of the normalised strengths of elements 1-3, for stiffness and strength proportional models (a), and torsionally asymmetric models (b), for systems designed by the MRSM. Part (c) indicates the corresponding variation of the normalised base shear force BSF. centre of the structure) with increasing $e_{s}$, shown in Figure 5(b) for the regular structure only. Despite the decreasing stiffness of the flexible-edge element 3 with increasing eccentricity (evident from Figure 5(a)), its design strength for the scaled results in Figure 5(b) remains almost constant at between $31 \%$ and $38 \%$ of the SBSF.

The combined inelastic base shear force derived from the MRSM, normalised to the SBSF, is shown in Figure 5(c). This quantity is affected across the full range of $e_{s}$ by the $100 \%$ scaling factor $\left(\mathrm{S}_{\mathrm{m} 2}, \mathrm{~K}_{\mathrm{m}}=1.0\right)$ applicable to horizontally irregular structures, and for eccentricities $e_{s} / b \geq 0.03$ by the $80 \%$ scaling factor $\left(\mathrm{K}_{\mathrm{m}}=0.8\right)$ for regular structures. With the scaling factor $\mathrm{S}_{\mathrm{m} 2}$ neglected, the combined inelastic base shear force derived from the MRSM decreases with increasing eccentricity, to a value of about $60 \%$ of the SBSF when $\mathrm{e}_{\mathrm{s}} / \mathrm{b}=$ 0.35 . As commented above, this proportion may be even lower in torsionally flexible buildings with $\Omega<1.0$.

\section{Element strength ratios}

By normalising the element strengths for each asymmetric model $\left(e_{s}>0\right)$, including the appropriate code allowance for torsional effects, to the corresponding strengths of the stiffness and strength proportional model, element strength ratios have been derived (see Figure 6). These ratios give a useful indication of the allowance for torsional effects made by the code. The element strength ratios plotted in Figure 6, corresponding to systems designed by the MRSM, have been scaled using the factor for horizontally-regular structures stipulated by NZS 4203:1992 (the combined inelastic base shear force derived from the MRSM equals $80 \%$ of the SBSF, hence $\mathrm{K}_{\mathrm{m}}=0.8$ ). Comparison is made with the corresponding strength ratios resulting from application of the code static torsional provisions [11]. It is noted that both methods result in similar reductions in the strength of the stiff-edge element 1 with increasing $e_{s}$. This reduction is particularly evident for buildings with intermediate or large static eccentricities. For example, a 


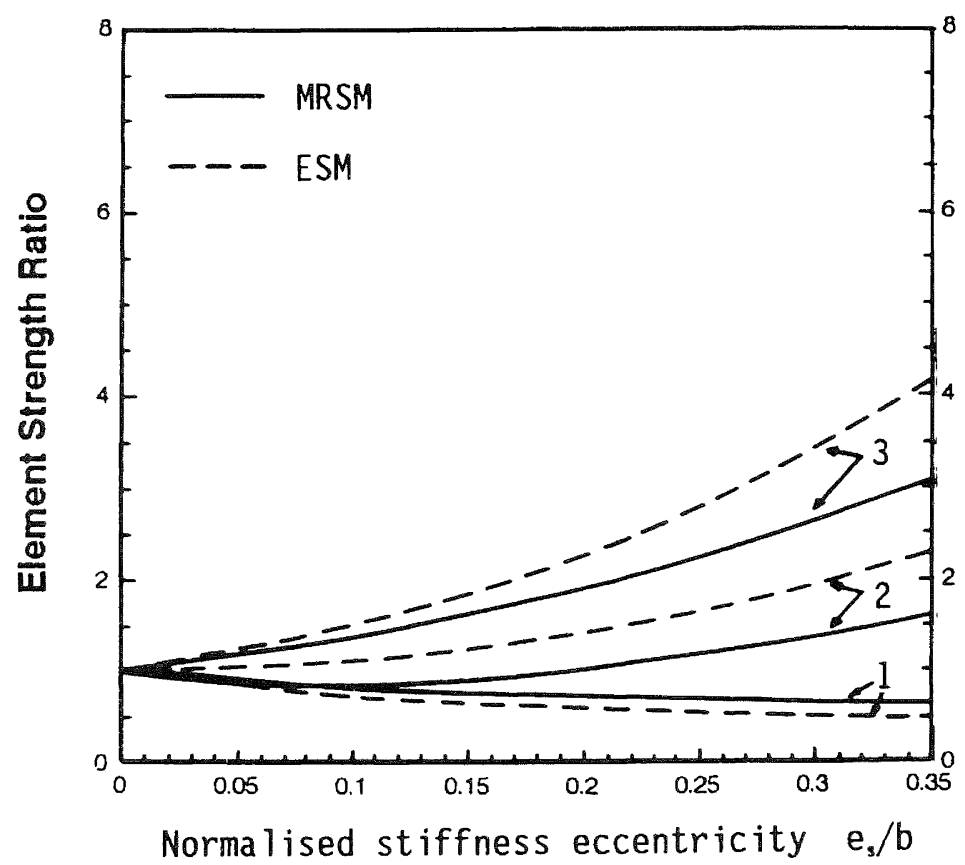

Figure 6. Distribution of strength ratios for elements 1-3 based on design by the MRSM and ESM (NZS 4203:1992, horizontally regular structures, $K_{m}=0.8$ )

maximum of about $50 \%$ reduction in element 1 strength arises in buildings with $e_{s} / b=0.35$, designed using the NZS 4203:1992 static torsional provisions, whereas the MRSM leads to a corresponding reduction of about $40 \%$.

Element 2 (at the centre of the building), and to a greater extent element 3 , experiences increases in strength when either design method is employed, intended to account both for larger elastic strength demand and the additional inelastic deformation of elements on the more flexible side of the structure. Related studies of code-designed structures $[10,11]$ have shown that in both the elastic and inelastic ranges of response, the flexible-edge displacements may be up to 3 or 4 times greater (compared with symmetric cases) in highly asymmetric systems with intermediate or low torsional stiffness $(\Omega \leq 1.0)$, and that this displacement ratio is not affected significantly, in the inelastic range, by the element design strength. It is observed from Figure 6 that both design methods result in significant increases in the design strength (and hence the yield displacement) of element 3 , for example, by ratios of 3.1 (MRSM), and $4.2(E S M)$, when $e_{s} / b=0.35$. These values are of the same order as the estimated increases in element deformation during inelastic earthquake response, as quoted above. This implies that the design strength of the flexible-edge element should be conservatively estimated by both the MRSM and the ESM. The inelastic performance of torsionally asymmetric systems designed by the MRSM has been evaluated below.

\section{Strength eccentricity}

The strength eccentricity $\left(e_{p}\right.$, Figure 1$)$ has been found [6] to be an influential parameter in the inelastic torsional response of asymmetric buildings, and its value has been determined based on the MRSM and the ESM, the latter using the static torsional provisions of NZS 4203:1992. When accidental eccentricity effects are neglected, the design static lateral shear force (=
SBSF) is applied through CM. The resulting plastic centroid PC (or centre of strength) is located at CM, and the corresponding strength eccentricity is therefore equal to $\mathrm{e}_{s}$. For most codes, the application of the static torsional provisions results in $\mathrm{PC}$ being located close to $C M[18]$, and hence $e_{p} \approx e_{s}$. It is notable that the distribution of element strengths resulting from the MRSM [Figure 5(b)] leads to relatively low values of strength eccentricity $e_{p}$, with PC located roughly mid-way between $C S$ and $C M$, moving slightly closer to $C M$ when the eccentricity is large.

\section{ASYMMETRIC BUILDINGS DESIGNED BY THE MRSM: INELASTIC RESPONSE}

\section{Solution of equations of motion}

The equations of motion of the single-storey building model have been solved for the purposes of inelastic response analyses using the step-by-step numerical integration method. The time interval of the numerical integration is taken to be $1 / 25$ of the shorter of the two initial elastic natural periods, or the time increment of digitisation of the earthquake input record if smaller. The numerical calculation has been carried out using the computer program DRAIN-TABS [19].

\section{Inelastic response parameter}

The design philosophy of codes, for structures responding inelastically under severe earthquake loading, is based on the limitation to acceptable values of the peak displacement ductility demand and peak deformation of the resisting elements. One example is the force reduction factor employed by several codes [20], which is an indicator of the global or system ductility of structures, and is different from the local or member ductility. The analogous parameter in NZS 4203:1992 is the structural ductility factor, $\mu$. 
(a) El Centro SOOE

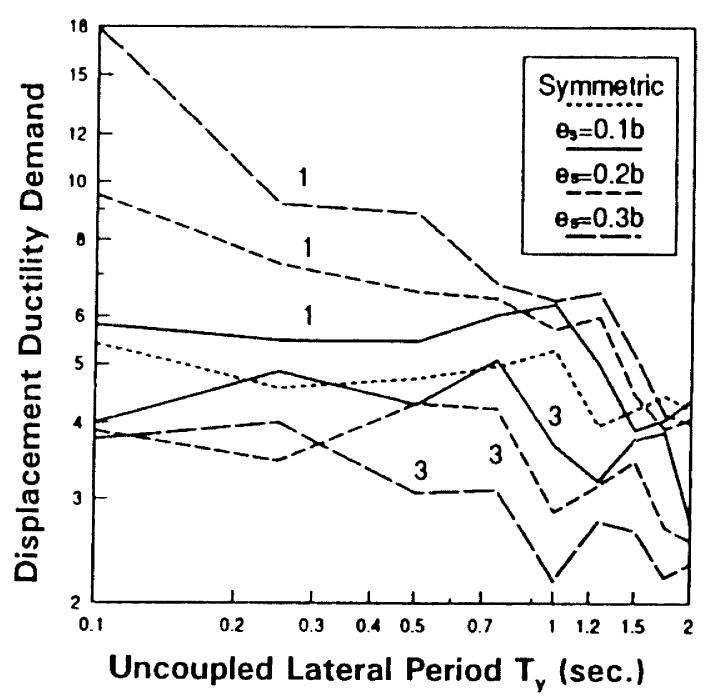

(b) 3470 Wilshire Blvd. NOOE

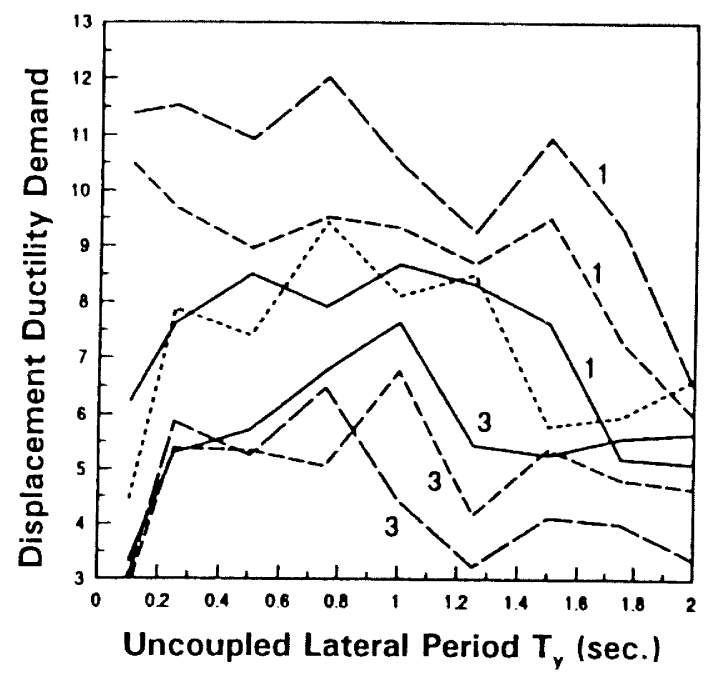

Figure 7. Peak inelastic displacement ductility demand of edge resisting elements 1 and 3 of asymmetric systems designed in accordance with the MRSM of NZS 4203:1992 (horizontally irregular structures, $K_{m}=1.0$ ), compared with reference symmetric cases.

For single-degree-of-freedom structures, the system ductility can be related directly to $\mu$ [21], but for multistorey buildings this relationship is more complex. Another example is the maximum allowable storey drifts specified in seismic codes. These provisions also represent a form of displacement ductility limit, in the sense that the deformation is restricted to a maximum value. Therefore, the present study employs the peak displacement ductility demand as the characteristic inelastic earthquake response parameter of the resisting elements.

\section{Earthquake ground motions}

Two records selected from Californian strong-motion earthquakes recorded at intermediate (moderately stiff) soil sites have been utilised as ground motion input for the assessment of the MRSM for inelastic (ultimate limit state) seismic structural design of asymmetric structures. The records consist of the Imperial Valley 1940 earthquake, El Centro SOOE record, and the San Fernando 1971 earthquake, 3470 Wilshire Blvd. N00E record.

These records have been appropriately scaled to a common peak ground acceleration of $0.32 \mathrm{~g}$. As shown in Figure 7 , this scaling results in displacement ductility demands for symmetric systems that are close to the magnitude of the structural ductility factor, $\mu=6$. The elastic response spectra of these records reflect their differing frequency content, as discussed in [22]. Since the aim of this study is to observe the response characteristics of asymmetric buildings with element strengths defined by the MRSM, and to make some qualitative comparisons with the results of related studies [10],[11] considering the performance of buildings designed by static code torsional procedures (ESM), rather than to obtain specific numerical responses or to treat the results in a statistical manner, the use of these two representative strong motion earthquake records is considered to be justified.

\section{Inelastic torsional effects}

The peak ductility demand for the edge elements 1 and 3 in asymmetric buildings having small, intermediate and large eccentricities, $e_{s}=0.1 b, 0.2 b$ and $0.3 b$, respectively, is presented in Figures 7(a),(b). Also shown, for purposes of comparison, are the responses of the corresponding reference symmetric systems with $e_{\varsigma}=0$ and uniformly distributed stiffness and strength. The structures analysed $\left(e_{\varsigma} \leq 0.3 \mathrm{~b}\right)$ are all categorised according to NZS 4203:1992 as horizontally regular, and hence in determining the element design strengths the scaling factor $\mathrm{K}_{\mathrm{m}}$ has been assigned the value 0.8 .

The results in Figure 7 indicate clearly that the stiff-edge element (element 1) is the critical element experiencing much higher inelastic response than the reference symmetric system, and that correspondingly the displacement ductility demand of the element at the flexible edge (element 3 ) is much lower than that of the reference system. Comparisons that have been made [22] between these results and those of related studies of inelastic torsional effects in buildings designed by code static procedures [6,8-11] indicate that the MRSM and the ESM result in the same qualitative and similar quantitative inelastic performance of asymmetric buildings.

Figure 8 presents the normalised displacement ductility for the edge elements 1 and 3. This is defined as the ratio of the peak ductility demand in asymmetric systems to that in the corresponding element in reference symmetric systems. Values of normalised displacement ductility greater than 1.0 indicate that torsional effects give rise to additional ductility demand, and hence increased likelihood of damage, in asymmetric systems. It is observed from Figure 8 that the MRSM may lead to satisfactory inelastic performance of asymmetric buildings having a small eccentricity $\left(\mathrm{e}_{\mathrm{s}}=0.1 \mathrm{~b}\right.$, for example), but results in relatively poor inelastic performance of those asymmetric buildings having intermediate to large eccentricities $\left(e_{s} \geq 0.2 b\right)$. 
(a) El Centro SOOE

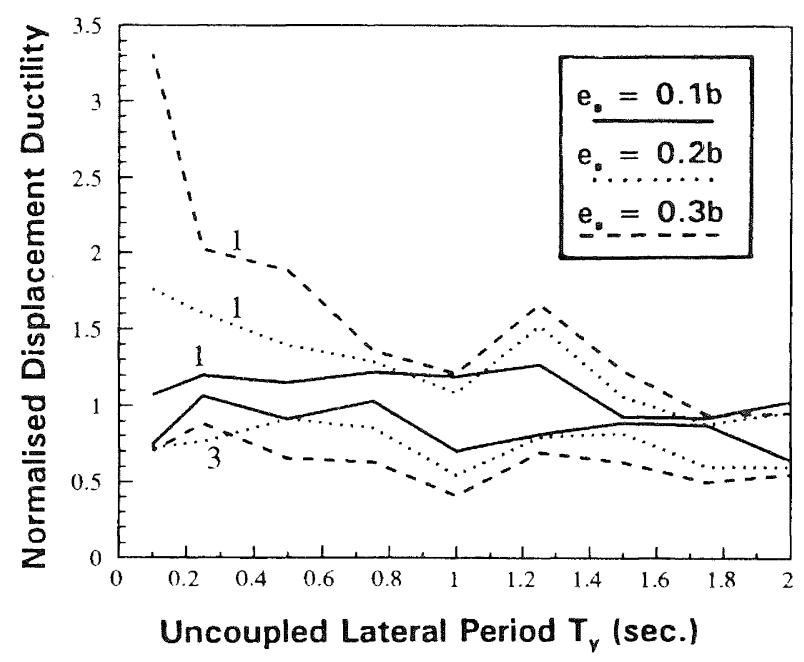

(b) 3470 Wilshire Blvd. NOOE

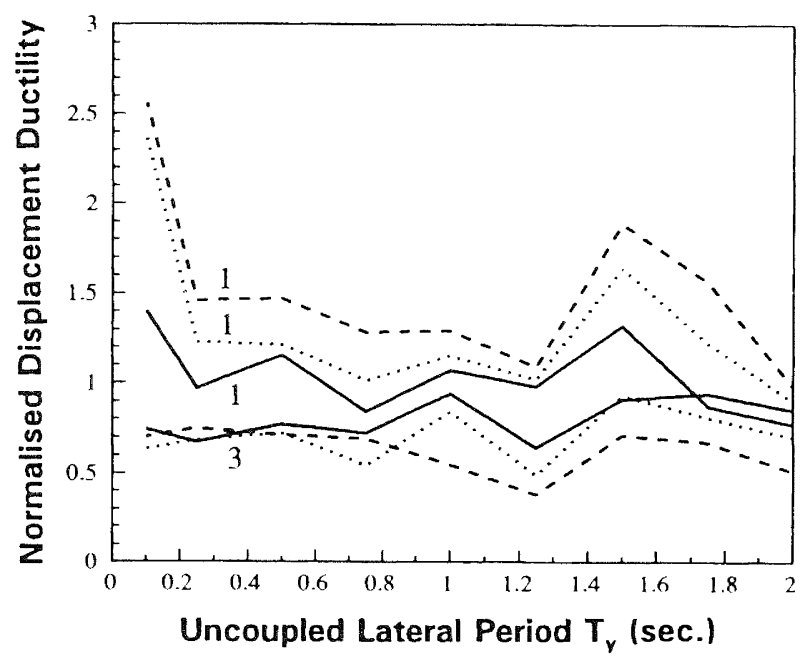

Figure 8. Normalised peak ductility demand of edge elements 1 and 3 of asymmetric systems designed by the MRSM of NZS 4203:1992.

This is attributed to the reduction of element 1 strength compared with the corresponding stiffness and strength proportional systems, which is inherent in the elastic spectral modal analysis design procedure (see Figure 6). This reduction leads to an underestimation of strength demand for the stiff-edge resisting element. The results presented in Figure 8 indicate that this underestimation occurs across the full period range $\left(T_{y}\right)$ considered, namely up to 2.0 seconds, and follows a similar pattern for both the selected earthquake records. The additional ductility demand in element 1 increases significantly with the magnitude of the eccentricity, $e_{s}$. The mean additional ductility demand for buildings with small eccentricity, $e_{s}=0.1 b$, has been calculated as about 1.15 , representing a $15 \%$ increase compared with symmetric systems. Whilst this may be considered acceptable, for buildings with $\mathrm{e}_{\mathrm{s}}=0.3 \mathrm{~b}$ the additional ductility demand has significantly higher values, calculated to be between $50 \%$ and $100 \%$, for medium-period and long-period systems.

In contrast, the results show clearly that the MRSM gives conservative control of peak ductility demand in the flexible-edge element (element 3 ) over the full range of eccentricities and periods considered. In fact, this provision may be regarded as somewhat over-conservative for buildings with intermediate or large $e_{s}$. For example, medium-period or long-period systems may have peak ductility demands as low as 2 or 3 (Figure 7). This is due to the allowance for significantly increased strength in this element, compared with the stiffness and strength proportional system. This increase accounts mainly for lateral-torsional coupling effects in the elastic range of response. Nevertheless, since the yield displacement of this element has been increased in accordance with its increased strength, the peak deformation of elements at the flexible edge of the building may still be very high compared with symmetric, elastic systems (as discussed above), due to a combination of the torsional effect and inelastic response.

The results shown in Figures 7 and 8 illustrate the difficulty arising in asymmetric structures when attempting to specify element strength distributions which satisfy both elastic and inelastic design criteria with reasonable (and consistent) levels of conservatism. This problem has still to be satisfactorily resolved, with regard to the performance of both the flexible-edge element (which is the critical element with regard to elastic deformation and corresponding strength demand) and the stiff-edge element (which is the critical element with regard to additional inelastic ductility demand). These issues are the subject of on-going research studies.

It is concluded that using the MRSM (as in NZS 4203:1992) to account for torsional effects, may lead to element strength distributions for systems with intermediate or large stiffness eccentricity $\left(e_{s} \geq 0.2 b\right)$ which do not satisfactorily achieve the design aim in the ultimate limit state of consistent protection given to symmetric and asymmetric buildings against excessive inelastic response and consequent structural damage.

\section{CONCLUSIONS AND RECOMMENDATIONS}

Design procedures for torsional effects should consider a number of factors which are, with present knowledge, difficult both to predict and to evaluate. These include the influence of lateral and torsional frequencies, the importance of adequate design of vertical resisting elements on both sides of the centre of stiffness, accidental eccentricity effects due to a variety of causes, and inelastic structural response. The last of these effects has been found to have a significant influence on the torsional structural response to severe earthquakes, fundamentally altering the dynamic response behaviour compared with systems responding in the elastic range. This paper has evaluated the horizontal regularity requirements and the MRSM of the 1992 edition of the New Zealand seismic loadings code, by inelastic dynamic analysis of an idealised but representative asymmetric building model. The following are the main conclusions and recommendations:

(1) The MRSM leads to an element strength distribution for systems with intermediate or large eccentricity $\left(e_{s} \geq 0.2 b\right)$ which does not satisfactorily achieve the design aim of consistent protection for symmetric and asymmetric buildings against excessive inelastic response and consequent 
structural damage. This is apparent, even when the yielding strengths of resisting elements are scaled up such that the combined inelastic base shear force derived from the MRSM is equal to $80 \%$ of the SBSF obtained from the ESM.

(2) The main cause of the above result is that the MRSM results in significantly reduced design strengths for stiff-edge elements (by up to $40 \%$ for systems with large eccentricity), compared with corresponding stiffness- and strengthproportional systems with no consideration of torsional effects. As a result, this design method may underestimate by a substantial amount the strength demand of such elements, leading to excessive additional ductility demand in highly asymmetric systems, compared with symmetric systems. In contrast, for flexible-edge elements, the peak ductility demand is usually substantially lower than that of the corresponding symmetric systems.

(3) From this and earlier studies, the equivalent static and spectral modal analysis methods (in their present forms) have been found to give satisfactory results only when applied to buildings with relatively small eccentricity. For buildings with $\mathrm{e}_{\mathrm{s}}=0.1 \mathrm{~b}$, for example, the additional ductility demand in the critical stiff-edge element has been found to be controlled to an acceptable level by both methods; hence this may represent an appropriate limit for their application in torsional design. Further results are required, however, to assess the additional ductility demand in more detail and hence to develop reliable design guidelines. Based on the results presented herein, it is tentatively recommended that any beneficial effects of torsion on element strength demand (particularly affecting elements on the stiff side of $\mathrm{CR}$ ) should be neglected for systems with static eccentricity greater than $0.1 \mathrm{~b}$. It is also recommended that for both regularly asymmetric and irregularly asymmetric buildings, the combined inelastic base shear force derived from the MRSM be at least equal to the SBSF obtained using the ESM. This recommendation is consistent with the regulation stipulated in the 1990 edition of the National Building Code of Canada.

(4) The second of the two horizontal regularity criteria recommended in NZS 4203: 1992, which is intended to limit floor rotations, does not appear to permit any structures to be designed by the ESM that are excluded by the first requirement, namely structures with $e_{s} \geq 0.3 b$. Hence it is concluded that the second criterion, which in its present form is much more restrictive than the first criterion, is effectively redundant for all practical cases.

(5) The results given in this paper illustrate the difficulty arising in asymmetric structures when attempting to satisfy both elastic and inelastic design criteria with reasonable (and consistent) levels of conservatism. This problem has still to be satisfactorily resolved, with regard to the performance of both the flexible-edge and stiff-edge elements, and is the subject of on-going research studies.

\section{ACKNOWLEDGEMENTS}

The authors gratefully acknowledge the support received from the British Council for the Ph.D research studies of Dr. X.N. Duan, carried out at University College London between 1988 and 1992, under the Technical Co-operation Training Scheme between the U.K. and the People's Republic of China. Investigations into inelastic torsional effects are continuing with the support of the UK Engineering and Physical Sciences Research Council (EPSRC), under Grant GR/J41062. This support is also gratefully acknowledged.

\section{REFERENCES}

1. Standards Association of New Zealand. 1992. Code of practice for general structural design and design loadings for buildings. NZS 4203. Wellington, New Zealand.

2. Cheung, V.W.-T. and Tso, W.K. 1986. Eccentricity in irregular multistorey buildings. Canadian Journal of Civil Engineering 13(1):46-52.

3. Jiang, W., Hutchinson, G.L. and Chandler, A.M. 1993. Static eccentricity for design of asymmetric shear buildings. Engineering Structures 15(3):167-178.

4. Chandler, A.M. and Hutchinson, G.L. 1987. Evaluation of code torsional provisions by a time history approach. Earthquake Engineering and Structural Dynamics 15(4):517-536.

5. Chandler, A.M. and Hutchinson, G.L. 1988. A modified approach to earthquake resistant design of torsionally coupled buildings. Bulletin of the New Zealand National Society for Earthquake Engineering 21(2): 140-153.

6. Chopra, A.K. and Goel, R.K. 1991. Evaluation of torsional provisions in seismic codes. Journal of Structural Engineering. ASCE 117(12):3762-3782.

7. Duan, X.N. 1991. Inelastic response and effective design of asymmetric buildings under strong earthquake loading. Ph.D thesis, University of London.

8. Rutenberg, A., Eisenberger, M. and Shohet, G. 1992. Inelastic seismic response of code-designed single storey asymmetric structures. Engineering Structures 14(2):91-102

9. Tso, W.K. and Ying, H. 1992. Lateral strength distribution specification to limit additional inelastic deformation of torsionally unbalanced structures. Engineering Structures 14:263-277.

10. Tso, W.K. and Zhu, T.J. 1992. Design of torsionally unbalanced structural systems based on code provisions I: ductility demand. Earthquake Engineering and Structural Dynamics 21:609-627.

11. Correnza, J.C., Hutchinson, G.L. and Chandler, A.M. In Press. Seismic response of flexible-edge elements in code-designed torsionally unbalanced structures. Engineering Structures. 
12. Tsicnias, T.G. and Hutchinson, G.L. 1981. Evaluation of code requirements for the earthquake resistant design of torsionally coupled buildings. Proceedings of the Institution of Civil Engineers. London 71(2):821-843.

13. Tso, W.K. and Dempsey, K.M. 1980. Seismic torsional provisions for dynamic eccentricity. Earthquake Engineering and Structural Dynamics 8:275-289.

14. Chandler, A.M. and Duan, X.N. 1991. Evaluation of factors influencing the inelastic seismic performance of torsionally asymmetric buildings. Earthquake Engineering and Structural Dynamics 20(1):87-95.

15. Duan, X.N. and Chandler, A.M. In Press. Reply to Discussion by W.K. Tso, Earthquake Engineering and Structural Dynamics.

16. Chandler, A.M., Correnza, J.C. and Hutchinson, G.L. In Press. Influence of code accidental eccentricity on inelastic seismic torsional effects in buildings. Engineering Structures.

17. Maison, B.F. and Neuss, C.F. 1983. SUPER-ETABS: an enhanced version of the ETABS program. A report to the National Science Foundation, J.G. Bouwkamp Inc., Berkeley, California, USA.

18. Tso, W.K. and Ying, H. 1990. Additional seismic inelastic deformation caused by structural asymmetry. Earthquake Engineering and Structural Dynamics 19:243-258.

19. Guendelman-Israel, R. and Powell, G.H. 1977. DRAIN-TABS: a computerized program for inelastic earthquake response of three-dimensional buildings. EERC Report No.77-08, University of California, Berkeley.

20. Tso, W.K. and Naumoski, N. 1991. Evaluation of NBCC 90 seismic force reduction factors. Proceedings of the 6th Canadian Conference on Earthquake Engineering. Toronto 751-758.

21. Newmark, N.M. and Riddell, R. 1980. Inelastic spectra for seismic design. Proceedings of the 7th World Conference on Earthquake Engineering. Istanbul, Turkey 4:129-136.

22. Duan, X.N. and Chandler, A.M. 1993. Inelastic seismic response of code-designed multistorey frame buildings with regular asymmetry. Earthquake Engineering and Structural Dynamics 22:431-445. 